University of Nebraska - Lincoln

DigitalCommons@University of Nebraska - Lincoln

\title{
Conformational Sampling of the Botulinum Neurotoxin Serotype a Light Chain: Implications for Inhibitor Binding
}

James C. Burnett

Developmental Therapeutics Program, NCI Frederick, Frederick, MD 21702, USA

James J. Schmidt

US Army Medical Research Institute of Infectious Diseases, Frederick, MD 21702, USA

Connor F. McGrath

Developmental Therapeutics Program, NCI Frederick, Frederick, MD 21702, USA

Tam L. Nguyen

Developmental Therapeutics Program, NCI Frederick, Frederick, MD 21702, USA

Ann R. Hermone

Developmental Therapeutics Program, NCI Frederick, Frederick, MD 21702, USA

See next page for additional authors

Follow this and additional works at: https://digitalcommons.unl.edu/usarmyresearch

Part of the Operations Research, Systems Engineering and Industrial Engineering Commons

Burnett, James C.; Schmidt, James J.; McGrath, Connor F.; Nguyen, Tam L.; Hermone, Ann R.; Panchal, Rekha G.; Vennerstrom, Jonathan L.; Kodukula, Krishna; Zaharevitz, Daniel W.; Gussio, Rick; and Bavari, Sina, "Conformational Sampling of the Botulinum Neurotoxin Serotype a Light Chain: Implications for Inhibitor Binding" (2005). US Army Research. 19.

https://digitalcommons.unl.edu/usarmyresearch/19

This Article is brought to you for free and open access by the U.S. Department of Defense at DigitalCommons@University of Nebraska - Lincoln. It has been accepted for inclusion in US Army Research by an authorized administrator of DigitalCommons@University of Nebraska - Lincoln. 


\section{Authors}

James C. Burnett, James J. Schmidt, Connor F. McGrath, Tam L. Nguyen, Ann R. Hermone, Rekha G.

Panchal, Jonathan L. Vennerstrom, Krishna Kodukula, Daniel W. Zaharevitz, Rick Gussio, and Sina Bavari 


\title{
Conformational sampling of the botulinum neurotoxin serotype a light chain: implications for inhibitor binding
}

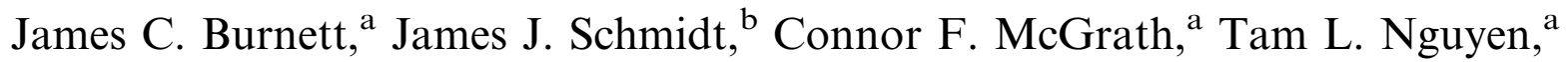 \\ Ann R. Hermone, ${ }^{\mathrm{a}}$ Rekha G. Panchal, ${ }^{\mathrm{a}}$ Jonathan L. Vennerstrom, ${ }^{\mathrm{c}}$ Krishna Kodukula, ${ }^{\mathrm{d}}$ \\ Daniel W. Zaharevitz, ${ }^{a}$ Rick Gussio ${ }^{\mathrm{a}, *}$ and Sina Bavari ${ }^{\mathrm{b}, *}$ \\ ${ }^{a}$ Developmental Therapeutics Program, NCI Frederick, Frederick, MD 21702, USA \\ ${ }^{\mathrm{b}}$ US Army Medical Research Institute of Infectious Diseases, Frederick, MD 21702, USA \\ ${ }^{\mathrm{c}}$ College of Pharmacy, University of Nebraska Medical Center, 986025 Nebraska Medical Center, Omaha, NE 68198, USA \\ ${ }^{\mathrm{d}}$ Office of Technology and Strategy, Innovation and Acquisitions, Sarnoff Corporation, 201 Washington Road, \\ Princeton, NJ 08543, USA
}

Received 19 August 2004; revised 9 October 2004; accepted 9 October 2004

Available online 11 November 2004

\begin{abstract}
Botulinum neurotoxins (BoNTs) are the most potent of the known biological toxins, and consequently are listed as category A biowarfare agents. Currently, the only treatments against BoNTs include preventative antitoxins and long-term supportive care. Consequently, there is an urgent need for therapeutics to counter these enzymes - post exposure. In a previous study, we identified a number of small, nonpeptidic lead inhibitors of BoNT serotype A light chain (BoNT/A LC) metalloprotease activity, and we identified a common pharmacophore for these molecules. In this study, we have focused on how the dynamic movement of amino acid residues in and surrounding the substrate binding cleft of the BoNT/A LC might affect inhibitor binding modes. The X-ray crystal structures of two BoNT/A LCs (PDB refcodes = 3BTA and 1E1H) were examined. Results from these analyses indicate that the core structural features of the examined BoNT/A LCs, including $\alpha$-helices and $\beta$-sheets, remained relatively unchanged during $1 \mathrm{~ns}$ dynamics trajectories. However, conformational flexibility was observed in surface loops bordering the substrate binding clefts in both examined structures. Our analyses indicate that these loops may possess the ability to decrease the solvent accessibility of the substrate binding cleft, while at the same time creating new residue contacts for the inhibitors. Loop movements and conformational/positional analyses of residues within the substrate binding cleft are discussed with respect to BoNT/A LC inhibitor binding and our common pharmacophore for inhibition. The results from these studies may aid in the future identification/development of more potent small molecule inhibitors that take advantage of new binding contacts in the BoNT/A LC.

Published by Elsevier Ltd.
\end{abstract}

\section{Introduction}

Botulinum neurotoxins (BoNTs) are secreted by anaerobic spore-forming bacteria Clostridium botulinum, and are the most poisonous of known biological substances. ${ }^{1}$ It is estimated that the lethal intravenous dose of BoNT

\footnotetext{
Abbreviations: BoNT, botulinum neurotoxin; BoNT/A, botulinum neurotoxin serotype A; BoNT/B, botulinum neurotoxin serotype B; LC, light chain; HC, heavy chain; Rmsd, rms deviation.

Keywords: Bioterrorism; Botulinum neurotoxin; Drug discovery; Inhibitors; Molecular dynamics; Molecular modeling; Pharmacophore; Metalloprotease.

* Corresponding authors. Tel.: +1 301846 5791; fax: +1 3018466106 (R.G.); tel.: +1 301619 4246; fax: +1 3016192348 (S.B.); e-mail addresses: gussio@ncifcrf.gov; sina.bavari@amedd.army.mil
}

serotype A (BoNT/A) in humans is $1-5 \mathrm{ng} \mathrm{kg}^{-1} \cdot 2,3$ Annually, numerous cases of accidental BoNT poisoning are reported in both humans and animals. ${ }^{1}$ Furthermore, as BoNTs continue to gain popularity as: (1) therapies for muscle hyperactivity and spasticity ${ }^{4-7}$ and (2) preferred agents for a range of cosmetic applications, ${ }^{8-10}$ inadvertent overdosing (requiring immediate toxin inhibition) may become problematic. More ominous is the fact that BoNTs have been weaponized in highly toxic aerosol form, and malevolent airborne release and/or food contamination pose a significant threat to both civilians and military personnel. ${ }^{2,11}$ In light of the lethality of BoNTs, and the longevity of the paralysis induced by these enzymes, ${ }^{12}$ there is a pressing need for a better understanding of how inhibitors might interact with these toxins. 
BoNTs are composed of a heavy chain (HC) $(100 \mathrm{kDa})$ and a light chain (LC) $(50 \mathrm{kDa})$, which are linked by a disulfide bridge. ${ }^{13}$ The $\mathrm{HC}$ is responsible for: (1) binding to surface receptors on cholinergic nerve terminals; (2) plasma membrane penetration via receptor mediated endocytosis; and (3) toxin release into the nerve cytosol. ${ }^{14,15}$ For BoNT/A, a protective belt extending from the translocation domain of the $\mathrm{HC}$ wraps around the LC and shields the substrate binding cleft prior to neuronal internalization. ${ }^{16}$ The LC (also referred to as the catalytic domain) separates from the $\mathrm{HC}$ under the low $\mathrm{pH}$ conditions of the endosome, and acts as a zinc metalloprotease that cleaves SNARE (soluble NSF-ethylmaleimide-sensitive factor attachment protein receptor) proteins. ${ }^{14,15}$ SNARE proteins are involved in membrane fusion and the exocytosis of neurotransmitter containing vesicles. ${ }^{17}$ BoNT serotypes A and E cleave SNAP-25 (synaptosomal-associated protein of $25 \mathrm{kDa}$ ) ${ }^{18}$ serotypes B, D, F, and G cleave VAMP (vesicle-associated membrane protein) or synaptobrevin, ${ }^{19-22}$ and serotype $\mathrm{C} 1$ cleaves both SNAP-25 and syntaxin $1 .{ }^{23}$ Following BoNT LC mediated cleavage of SNARE proteins, acetylcholine release into neuromuscular synapses is compromised, and paralysis ensues.

In a recent report, ${ }^{24}$ we identified novel, small (nonpeptidic) molecule leads that inhibit BoNT/A LC metalloprotease activity by up to $62 \%$ at $20 \mu \mathrm{M}$ concentrations, and we developed a common pharmacophore for these inhibitors based on conformational analyses and molecular docking studies ${ }^{24}$ (Fig. 1 shows the two-dimensional structures of two of the most potent of these inhibitors, which will be referred to in the text). In this study, we used molecular dynamics to explore how the motion of residues in and around the BoNT/A LC substrate binding cleft might affect inhibitor binding. In general, the reported analyses support the crystallographically determined structures of BoNT/A LCs that were obtained from the holotoxin $(\mathrm{PDB} \text { refcode }=3 \mathrm{BTA})^{16}$ and $\mathrm{PDB}$ refcode $=1 \mathrm{E} 1 \mathrm{H}$ (two LCs engaged in intermolecular autocatalysis) ${ }^{25}$ $\alpha$-helices and $\beta$-sheets remained stable over the course of the dynamics simulations. However, our results do suggest that surface loops ${ }^{13,16,25}$ may reorient to par-

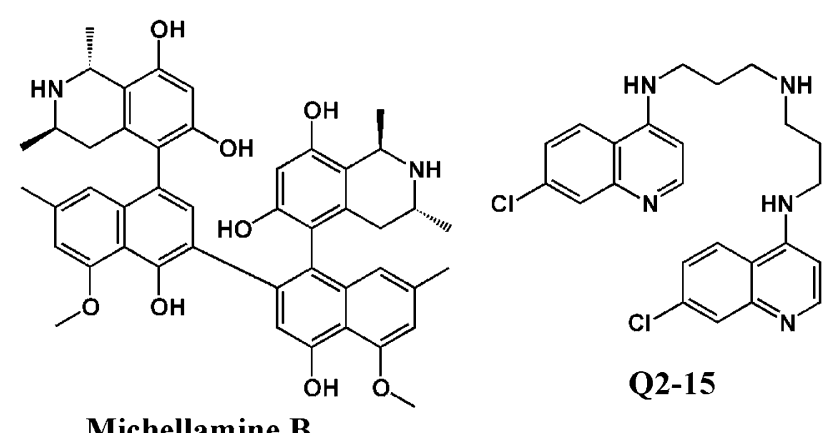

Figure 1. Two-dimensional structures of BoNT/A LC metalloprotease inhibitors michellamine B and Q2-15. Michellamine B potency: $62 \%$ inhibition at $20 \mu \mathrm{M}$ concn; Q2-15 potency: $60 \%$ inhibition at $20 \mu \mathrm{M}$ concn. tially shield the substrate binding cleft following LC release from the $\mathrm{HC}$, and that these loop movements may create new binding surfaces for inhibitors, and/or facilitate inhibitor desolvation. Molecular docking studies using previously identified small molecule inhibitors of BoNT/A LC metalloprotease activity ${ }^{24}$ have been used to refine our common pharmacophore. ${ }^{24}$

\section{Methods}

\subsection{Molecular modeling}

The BoNT/A holotoxin X-ray structure (PDB code $=$ $3 \mathrm{BTA}^{16}$ ) includes both the HC and the LC. First, the $\mathrm{HC}$, which is composed of the translocation domain (residues A448-A872) and the receptor binding domain (residues A873-A1295), was removed from the structure, as: (1) access to the substrate binding cleft of the BoNT/A is completely blocked by the protective belt of the $\mathrm{HC} ;{ }^{13,16}(2)$ it is the reduced form of this enzyme (i.e., the disulfide bridge connecting the $\mathrm{HC}$ and the LC is broken) that displays peptidase activity; ${ }^{19,26}$ and (3) the BoNT/A LC alone (i.e., without the HC component) is active. ${ }^{25,27}$ Hydrogens were added to the LC (residues $\mathrm{A} 1-\mathrm{A} 415$, which are complementary to the residues of the 1E1H BoNT/A LC structure (see below), ${ }^{25}$ ) and the structure was energy refined using the DISCOVER (Accelrys, San Diego, CA) program's cff91 force field. The zinc ion, and coordinating residues (H222, E223, H226, and E261) were fixed to their original crystallographic coordinates. The molecular mechanics energy refinement involved applying $2000 \mathrm{kcal} / \mathrm{mol}$ per $\AA^{2}$ of force that was stepped off the structure in $100 \mathrm{kcal} / \mathrm{mol}$ decrements by minimizing with conjugate gradients until the norm of the gradient was $0.01 \mathrm{kcal} / \AA^{2}{ }^{2}{ }^{28,29}$ The coordinates of the energy-refined model were within the experimentally determined X-ray crystallographic resolution, and were used as a starting point for molecular dynamics simulations.

Molecular dynamics were performed using DISCOver 3.0 , and involved $200 \mathrm{ps}$ of direct velocity scaling (cff91 force field, distance dependent dielectric, $0.5 \mathrm{fs}$ time step, initial temperature $=10 \mathrm{~K}$, final temperature $=300 \mathrm{~K}$ ), followed by dynamics using the Berendsen method of temperature-bath coupling ( $0.5 \mathrm{fs}$ time step; $300 \mathrm{~K}$ ) to $1 \mathrm{~ns}$. Initially, at least 100 lowest energy structures and 10 highest energy structures were used to analyze BoNT/A LC conformers collected over the course of the dynamics simulation.

The X-ray structure PDB code $=1 \mathrm{E} 1 \mathrm{H}^{25}$ is composed of two LCs engaged in the intermolecular autocatalysis of a peptide bond located in corresponding loops (composed of residues 232-258). For these studies, one of the LCs and all water molecules were removed from the X-ray crystal structure; the LC composed of residues A7-A249 and B251-B415 was chosen for dynamics simulations. Several residues were missing from surface loops of the crystal structure: L199, E200, V201, D202, T203, N204, P205, L206, Y250, and N393. These missing residues were built into the structure using 
corresponding residues in the 3BTA BoNT/A LC as templates. Following, hydrogens were added and the $1 \mathrm{E} 1 \mathrm{H}$ BoNT/A LC was subjected to the same molecular mechanics and molecular dynamics protocols as described for the 3BTA BoNT/A LC (see above). At least 100 lowest energy structures and 10 highest energy structures from the dynamics trajectory were used to analyze conformers of this BoNT/A LC.

Inhibitors were docked by combining molecular mechanics minimizations with the hydropathic scoring function HINT (eduSoft, Richmond, VA) in an iterative manner to achieve optimal complementarity with BoNT/A LC substrate binding clefts. ${ }^{28,29}$ With regard to BoNT/A LC dynamics structures, 3BTA structures collected up to 225 ps following trajectory equilibration were found to possess suitable complimentary for michellamine B docking; for $1 \mathrm{E} 1 \mathrm{H}$, structures collected up to $205 \mathrm{ps}$ following trajectory equilibration were found to possess suitable complementarity for docking Q215. Lowest energy structures from these trajectory ranges were subsequently used in the docking studies. For 3BTA, this corresponded to a structure collected at $188.5 \mathrm{ps}$; for $1 \mathrm{E} 1 \mathrm{H}$ this corresponded to a structure collected at $123.5 \mathrm{ps}$. These two structures were refined with molecular mechanics as described above. Following, compounds were manually docked into the BoNT/ A LC substrate binding clefts of the indicated dynamics models, as well as the molecular mechanics 'only' refined $\mathrm{X}$-ray crystal structures, and van der Waals violations $\geqslant 0.25 \AA$ were removed by small adjustments to inhibitor positioning and enzyme side-chain torsion angles. The inhibitor-enzyme structure coordinates were minimized in the same manner as described above, and were subjected to hydropathic analyses, using the program HINT, to eliminate/reduce unfavorable contacts. Coordinates for the docked models are available upon request.

\subsection{Compound information and HPLC-based assay}

Michellamine B (Fig. 1) ${ }^{24}$ was obtained from the National Cancer Institute, and Q2-15 (Fig. 1) ${ }^{24}$ was obtained from Dr. Jonathan Vennerstrom, University of Nebraska Medical Center. The HPLC based assay used to quantitate BoNT/A LC inhibition has been described in detail elsewhere. ${ }^{30,31}$

\section{Results and discussion}

Molecular dynamics simulations were used to evaluate BoNT/A LC conformations at $300 \mathrm{~K}$ over $1 \mathrm{~ns}$. These studies were initiated to answer the following question: how might residue movements in and around the BoNT/A LC substrate binding cleft affect predicted inhibitor binding modes, ${ }^{24}$ and how might this information be used to refine the common pharmacophore for BoNT/A LC inhibitors? ${ }^{24}$ Answers to this question will provide important information for ongoing studies to develop our lead inhibitors into therapeutically viable countermeasures against BoNT/A LC metalloprotease activity.

\subsection{Comparisons of BoNT/A LCs following dynamics simulations}

Two BoNT/A LC structures were examined. One BoNT/A LC was taken from the X-ray crystal structure of the holotoxin $\left(\mathrm{PDB}\right.$ refcode $\left.=3 \mathrm{BTA} ;{ }^{16}\right)$ the other was obtained from a recently released X-ray crystal structure of two LCs engaged in intermolecular autocatalysis $\left(\mathrm{PDB}\right.$ refcode $\left.=1 \mathrm{E} 1 \mathrm{H} .{ }^{25}\right)$ With regard to the dimeric 1E1H X-ray crystal structure, BoNT/A LC autocatalysis has been observed in solution, and may occur via an intermolecular route. ${ }^{32,33}$ Hence, these atomic coordinates $^{25}$ provide evidence to structurally explain a mechanism of intermolecular BoNT/A LC autocatalysis. $^{25,32,33}$

Following the dynamics simulations, analyses of at least 100 lowest-energy and 10 highest-energy structures from the trajectories of both BoNT/A LCs did not reveal the existence of significantly different conformations of this enzyme (Table 1). For example, individual superimpositions across all backbone atoms of each of the 100 lowest-energy conformers from the dynamics trajectory of the 3BTA BoNT/A LC with that of the average structure (from the same trajectory) resulted in a mean

Table 1. Comparisons of BoNT/A LC models

\begin{tabular}{|c|c|c|c|}
\hline \multirow[t]{2}{*}{ BoNT/A LC structures } & \multicolumn{3}{|c|}{$\mathrm{Rmsd}^{\mathrm{a}}$} \\
\hline & All $(\AA)$ & $\begin{array}{l}\text { Loops } 1-3 \\
\text { omitted }(\AA)\end{array}$ & $\begin{array}{l}\text { Only } \alpha \text {-helices } \\
\text { and } \beta \text {-sheets }(\AA)\end{array}$ \\
\hline \multicolumn{4}{|l|}{$3 B T A$} \\
\hline $\begin{array}{l}\text { Crystal versus } \\
\text { dynamics avg. }\end{array}$ & 4.0 & 2.7 & 1.8 \\
\hline $\begin{array}{l}\text { Dynamics avg. } \\
\text { versus } 100 \text { L.E confs. }\end{array}$ & $0.76^{\mathrm{d}}$ & & \\
\hline $\begin{array}{l}\text { Dynamics avg. } \\
\text { versus } 10 \text { H.E. confs. }{ }^{c}\end{array}$ & $0.86^{\mathrm{e}}$ & & \\
\hline \multicolumn{4}{|l|}{$1 E 1 H$} \\
\hline $\begin{array}{l}\text { Crystal versus } \\
\text { dynamics avg. }\end{array}$ & 3.8 & 2.4 & 1.8 \\
\hline $\begin{array}{l}\text { Dynamics avg. } \\
\text { versus } 100 \text { L.E confs. }\end{array}$ & $0.71^{\mathrm{f}}$ & & \\
\hline $\begin{array}{l}\text { Dynamics avg. versus } \\
10 \text { H.E. cons. }\end{array}$ & $0.73^{\mathrm{g}}$ & & \\
\hline \multicolumn{4}{|l|}{ ЗBTA versus $1 E 1 H$} \\
\hline Crystal versus crystal & 2.2 & 1.1 & 0.57 \\
\hline $\begin{array}{l}\text { Dynamics avg. versus } \\
\text { dynamics avg. }\end{array}$ & 3.8 & 2.4 & 1.7 \\
\hline
\end{tabular}

${ }^{a}$ All superimpositions were performed using backbone atoms.

${ }^{\mathrm{b}}$ L.E. confs. = lowest-energy conformers from the trajectory.

${ }^{\mathrm{c}}$ H.E. confs. $=$ highest-energy conformers from the trajectory.

${ }^{\mathrm{d}}$ The average rmsd for 100 L.E. confs. from the 3BTA dynamics trajectory compared with the average structure from the same trajectory $(\mathrm{rmsd}$ range $=0.64-0.94 \AA)$.

${ }^{\mathrm{e}}$ The average rmsd for 10 H.E. confs. from the 3BTA dynamics trajectory compared with the average structure from the same trajectory (rmsd range $=0.70-0.99 \AA$ )

${ }^{\mathrm{f}}$ The average rmsd for 100 L.E. confs. from the $1 \mathrm{E} 1 \mathrm{H}$ dynamics trajectory compared with the average structure from the same trajectory (rmsd range $=0.61-0.94 \AA$ ) .

${ }^{g}$ The average rmsd for 10 H.E. confs. from the $1 \mathrm{E} 1 \mathrm{H}$ dynamics trajectory compared with the average structure from the same trajectory (rmsd range $=0.63-0.79 \AA)$ 

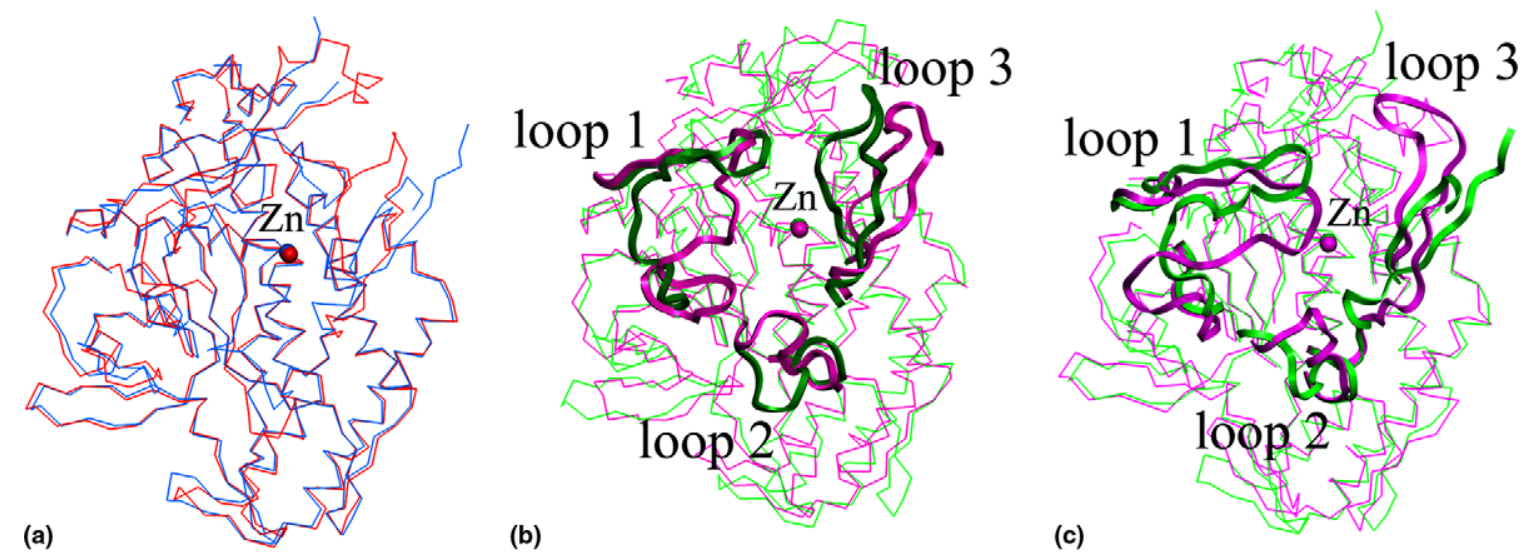

Figure 2. Comparisons of BoNT/A LC models. (a) All backbone atoms superimposition of the BoNT/A LCs from X-ray crystal structures PDB refcodes $=3 \mathrm{BTA}(\mathrm{red})$ and $1 \mathrm{E} 1 \mathrm{H}$ (blue); (b) all backbone atoms superimposition of the 3BTA BoNT/A LC X-ray crystal structure (green) and its dynamics average structure (magenta). Loops 1-3 are shown as ribbons; (c) all backbone atoms superimposition of the 1E1H BoNT/A LC X-ray crystal structure (green) and its dynamics average structure (magenta). Loops 1-3 are shown as ribbons.

rmsd of only $0.76 \AA$ (range $=0.61-1.0 \AA$ ), with a standard deviation of $0.95 \AA$ (Table 1). Comparable results were obtained when comparing lowest-energy conformers from the dynamics simulation of the $1 \mathrm{E} 1 \mathrm{H}$ BoNT/A LC with the average structure from its trajectory (Table 1). Additionally, the secondary structures (i.e., $\alpha$-helices and $\beta$-sheets) of the 3BTA and $1 \mathrm{E} 1 \mathrm{H}$ BoNT/A LC X-ray structures were nearly identical (Fig. 2a), and remained so throughout these analyses (Table 1).

\subsection{BoNT/A LC surface loop movements}

In contrast, the molecular dynamics studies did provide evidence that surface loops bordering the substrate binding clefts of the examined BoNT/A LCs possess the ability to undergo movement that may be important for optimizing inhibitor binding. The surface loops, ${ }^{13,25}$ referred to as: loop 1 (residues 48-78); loop 2 (residues 167-180); and loop 3 (residues 232-258) for the remainder of the text (Fig. 2b and c), were the main contributors to conformational differences between average structures obtained from the dynamics simulations and original X-ray crystallographic structures (Table 1). In accordance with these results, B-factors for residues forming $\alpha$-helices and $\beta$-sheets, which remained stable over time (as described above, Table 1), possess, in general, greater thermodynamically stability than residues in the indicated loops. Furthermore, the observed loop movements in both LC structures, which were solved under different crystallographic conditions, were very similar in nature. Segelke et al. ${ }^{25}$ and Lacy and Stevens ${ }^{13}$ have suggested that conformational changes in surface loops, which are also found in BoNT/B LC X-ray structures, ${ }^{34,35}$ may impact substrate recognition/binding and/or catalysis. The fact that our analyses indicate the possibility of conformational changes in these loops supports their hypotheses.

Observed loop 1 (residues 48-78) movements during dynamics simulations indicated that this structural feature may play an important role in inhibitor binding (Fig. $2 \mathrm{~b}$ and c). Interestingly, during the dynamics simu- lations of both the 3BTA and the 1E1H BoNT/A LCs, loop 1 possessed the ability to move toward and partially into the substrate binding clefts. Furthermore, it should be noted that the intermolecular lysis observed in the $1 \mathrm{E} 1 \mathrm{H}$ structure did not impact loop l's position in the X-ray crystal structure, and therefore would probably not play a significant role in predetermining the position of this loop.

Taking this information into consideration, the examined BoNT/A LC dynamics trajectories may provide snapshots of possible loop 1 movement. Specifically, the two X-ray crystal structures show loop 1 in a more 'open' conformation, allowing for unimpeded access to a solvent exposed cleft; however, dynamics simulations appear to indicate that the natural movement of loop 1 may allow it to reorient toward the LC substrate binding cleft, and it is this relatively more 'closed' conformation that may present additional enzyme contacts that facilitate inhibitor or substrate binding. At the same time, loop 1 movement partially shields the cleft from the solvent interface, which might aid the catalytic process. Future docking studies of the most potent BoNT/A LC inhibitor to date (a pseudo-peptide possessing a terminal 3-phenyl-2-thiol-propionyl attached via an amide bond to the N-terminus of RATKML $\left.\left(K_{\mathrm{i}}=300 \mathrm{nM}\right),{ }^{36}\right)$ and a rationalization of the SAR that accompanies this inhibitor, ${ }^{36}$ would help to clarify which conformation of the enzyme may be the active form.

Loop 2 (residues 167-180) in the X-ray structure of the 3BTA LC is oriented away from the substrate binding cleft - due to the presence of residues from the translocation domain protective belt (Fig. 2b). In the absence of the protective belt, this loop adopted a new conformation (i.e., during the dynamics simulations) that brought it into closer association with the substrate binding cleft. Similar orientation of loop 2 toward the substrate binding cleft was also observed for the $1 \mathrm{E} 1 \mathrm{H}$ LC (Fig. 2c). However, loop 2 in the 1E1H X-ray structure was positioned in such a way that it was already oriented toward the substrate binding cleft (as opposed to this loop in the 3BTA BoNT/A LC x-ray structure), thus 
providing further evidence that this loop is in closer association with the substrate binding cleft.

Loop 2 orientation toward the substrate binding cleft may: (1) provide additional ligand contacts and (2) decrease binding cleft solvent accessibility, which would reinforce inhibitor/substrate binding by creating a more favorable environment for desolvation. Indeed, it is possible that the combined movements of both loop 1 and loop 2 help induce the required conformation of the (otherwise flexible) substrate, SNAP 25, which is necessary for optimal binding. From a teleological perspective, perhaps taking advantage of such surface loop movements may have facilitated the evolution of highly toxic and substrate specific BoNT serotypes from a common ancestor.

Loop 3 (residues 232-258) movement during dynamics simulations of both examined BoNT/A LCs did not affect accessibility to the enzymes' substrate binding clefts. Both the 3BTA LC loop 3 and the 1E1H LC loop 3 collapsed toward space on the enzyme's surface (Fig. 2b and c) that was originally occupied by either: (1) the holotoxin translocation domain and its protective belt component (in the case of the 3BTA BoNT/A LC) or (2) the loop 3 of the opposing LC in the dimeric structure (in the case of the 1E1H BoNT/A LC).

\subsection{Loop movements and BoNT/A LC inhibitor binding}

In a previous report, ${ }^{24}$ molecular docking studies were used to define three regions in the BoNT/A LC substrate binding cleft that are hypothesized to be contact subsites for identified BoNT/A LC inhibitors. These regions included hydrophobic binding subsite 1 (composed of resi- dues F162, F177, F193, and T219), binding subsite 2 (composed of residues C164, T175, H226, R230, P238, and E260), and a more loosely organized polar contact region (composed of residues E55, Q161, E163, K165, and R176). During these earlier studies, a molecular mechanics refined BoNT/A LC from the 3BTA X-ray crystal structure served as the toxin model. ${ }^{24}$ Observed reorientations of BoNT/A LC surface loops bordering the enzyme's substrate binding cleft (during dynamics simulations) are now considered with respect to previously specified inhibitor contact subsites in both the $3 \mathrm{BTA}$ and the 1E1H BoNT/A LC models.

The composition of binding subsite 1 (also referred to as the $\mathrm{S}_{1}^{\prime}$ binding site ${ }^{25,36}$ ) remained relatively unchanged during the dynamics simulations, as examination of structures from the dynamics trajectories of both the 3BTA LC and the 1E1H LC showed that previously identified residues: F162, F187, F193, and T21924 composed this subsite consistently over time. Consequently, binding subsite 1 is a stable pocket where hydrophobic collapse between the enzyme and moieties from our inhibitors, such as a chloro, methyl, methoxy, or a dihydro imidazolyl, is likely to occur. ${ }^{24}$ For example, when one of our previously identified inhibitors, Q2-15 (Fig. 1) $(60 \%$ inhibition at $20 \mu \mathrm{M}$ concn $),{ }^{24}$ is docked into either a structure from the 1E1H BoNT/A LC dynamics trajectory (a low energy structure obtained at $123.5 \mathrm{ps}$ following trajectory equilibration), or the mechanics refined X-ray structure of $1 \mathrm{E} 1 \mathrm{H}$, one of the chloro substituents engages in favorable contacts with the hydrophobic side chains of residues in subsite 1 (Fig. 3a and b). At the same time, the accompanying quinoline nitrogen is in close proximity to the enzyme's catalytic engine, where it is positioned such that it may either engage in a
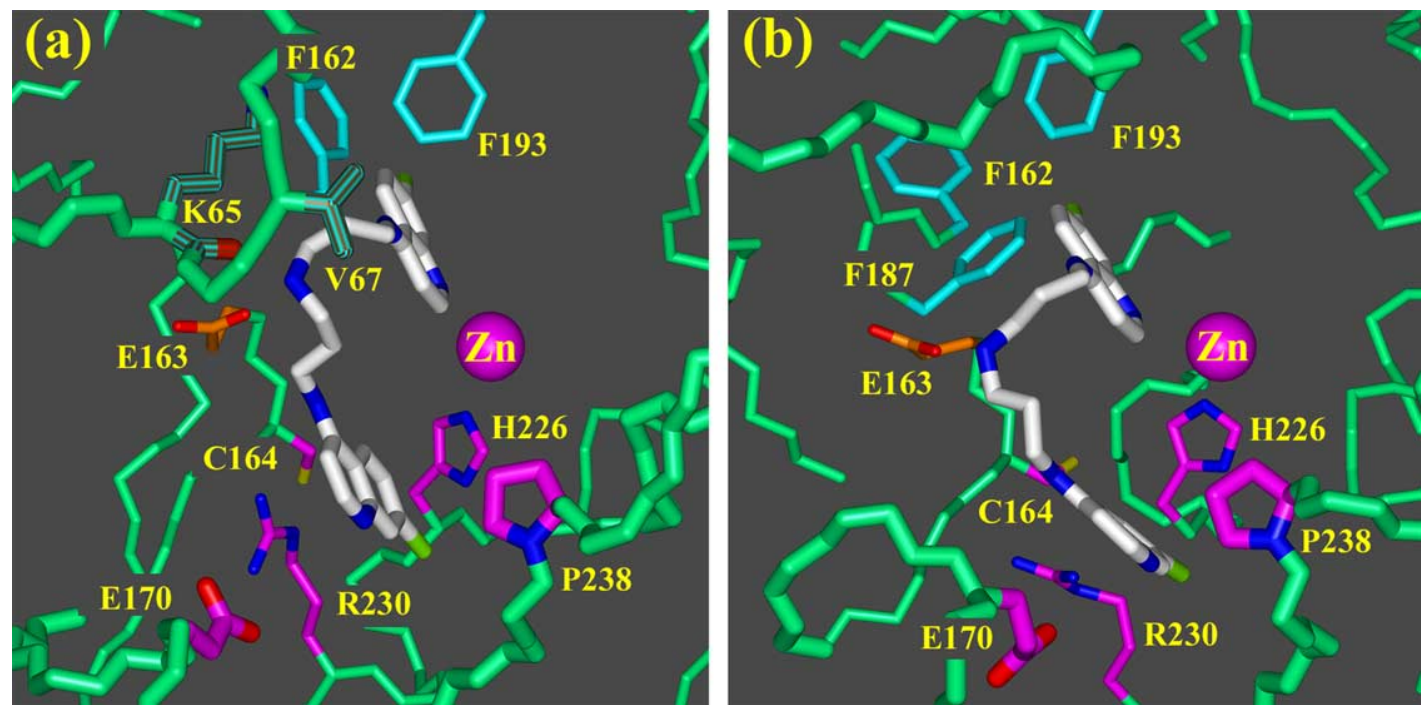

Figure 3. (a) BoNT/A LC inhibitor Q2-15 docked in the molecular dynamics model for the 1E1H BoNT/A LC. Enzyme atom colors: subsite 1 carbons (light blue); subsite 2 carbons (magenta); polar contact region carbons (orange); all other carbons (green); oxygen (red); nitrogen (blue); sulfur (yellow). Q2-15 carbons are white and chlorine atoms are light green. BoNT/A LC loops 1, 2, and 3, as well as the side chains of specified residues of these loops are shown in thicker stick. Residues with brown stripes are new contacts that are observed when docking Q2-15 in the dynamics BoNT/A LC (as opposed to contacts made by Q2-15 docked in the molecular mechanics refined X-ray structure); (b) BoNT/A LC inhibitor Q2-15 docked in the molecular mechanics refined X-ray crystal structure of the 1E1H BoNT/A LC. All colors and stick thickness are as described for (a). A comparison of (a) and (b) shows how loop 1 reorientation provides additional contacts for the inhibitor. 
direct interaction with the zinc ion, or displace the engine's catalytic water. In a similar manner, Figure 4a and $\mathrm{b}$ show how the methoxy and methyl substituents of michellamine B (Fig. 1) $(62 \%$ inhibition at $20 \mu \mathrm{M}$ concn) naphthalene ring A engage in favorable hydrophobic interactions with residues of binding subsite 1 in both a structure from the 3BTA dynamics simulation (a low energy structure obtained at $188.5 \mathrm{ps}$ following trajectory equilibration) and the mechanics refined X-ray structure of 3BTA, respectively. Additionally, as observed for the quinoline nitrogen of Q2-15, the hydroxyl moiety of naphthalene ring $\mathrm{A}$ is positioned such that it may interact directly with the enzyme's catalytic zinc, or displace the water that is used by the enzyme's catalytic engine.

The binding mode of Q2-15 in the dynamics structure of $1 \mathrm{E} 1 \mathrm{H}$ (Figure 3a) also shows that loop 1 orientation toward the enzyme's substrate binding cleft creates a new hydrophobic pocket near subsite 1 (composed of residues K65 (hydrophobic side chain), V67, and P68), which provides a complimentary binding surface for three methylene carbons that form half of the flexible linker connecting the inhibitor's two 7-chloroquinoline components (Fig. 3a). These additional hydrophobic contacts are not observed when Q2-15 is docked in the more 'open' mechanics refined structure (Fig. 3b). In like manner, michellamine B binding in the 3BTA dynamics structure (Fig. 4a) shows the methyl substituent of naphthalene ring $\mathrm{A}$ engaging in a hydrophobic contact with V67 (this contact is not observed when michellamine B is docked in the 3BTA mechanics refined structure (Fig. 4b)).

The solvent accessibility of binding subsite 2 is reduced during the dynamics simulation of the 3BTA structuredue to loop 2 movement (as indicated above, loop 2 was already oriented toward the substrate binding cleft in the $1 \mathrm{E} 1 \mathrm{H}$ X-ray crystal structure). However, these changes had little affect on the amino acids composing this subsite: C164, H226, R230, E260, and P238 remained key residues surrounding this pocket. Figure 3a and b show how Q2-15 binding within this subsite is very similar in both the $1 \mathrm{E} 1 \mathrm{H}$ dynamics structure and the $1 \mathrm{E} 1 \mathrm{H}$ mechanics refined X-ray structure, respectively. Specifically, the other Q2-15 chloroquinoline moiety docks in this subsite in such a way that the quinoline ring sits in close proximity to the side chain of R230 (with the quinoline nitrogen pointed toward that solvent interface), while the 7-chloro substituent of this moiety points into the binding subsite and engages in favorable hydrophobic contacts with the side-chain imidazole of $\mathrm{H} 226$ and the side-chain methylenes of E260.

Loop 2 reorientation is more dramatic when comparing michellamine B docked in the 3BTA dynamics structure (Fig. 4a) and the mechanics refined structure (Fig. 4b). Figure $4 \mathrm{a}$ shows how loop 2 reorientation increases the depth of this subsite - compared to the crystal structure (Fig. 4b) - with the entire loop rising as it moved toward the enzyme's binding cleft. As a result, a new boundary for this end of the substrate binding pocket is created, and solvent accessibility is reduced. With regard to michellamine B binding in subsite 2, the methoxy substituent of naphthalene ring B packs into space located behind H226 (in a manner similar to one of the chloro substituents of Q2-15) in both the 3BTA dynamics structure and the mechanics refined X-ray crystal structure. In contrast, the methyl substituent on naphthalene ring $B$ in the dynamics structure sits behind loop 2 and engages in hydrophobic contacts with the side-chain methylenes of residue E170 (Fig. 4a), while this same substituent in the molecular mechanics refined structure of 3BTA is more solvent exposed (Fig. 4b).
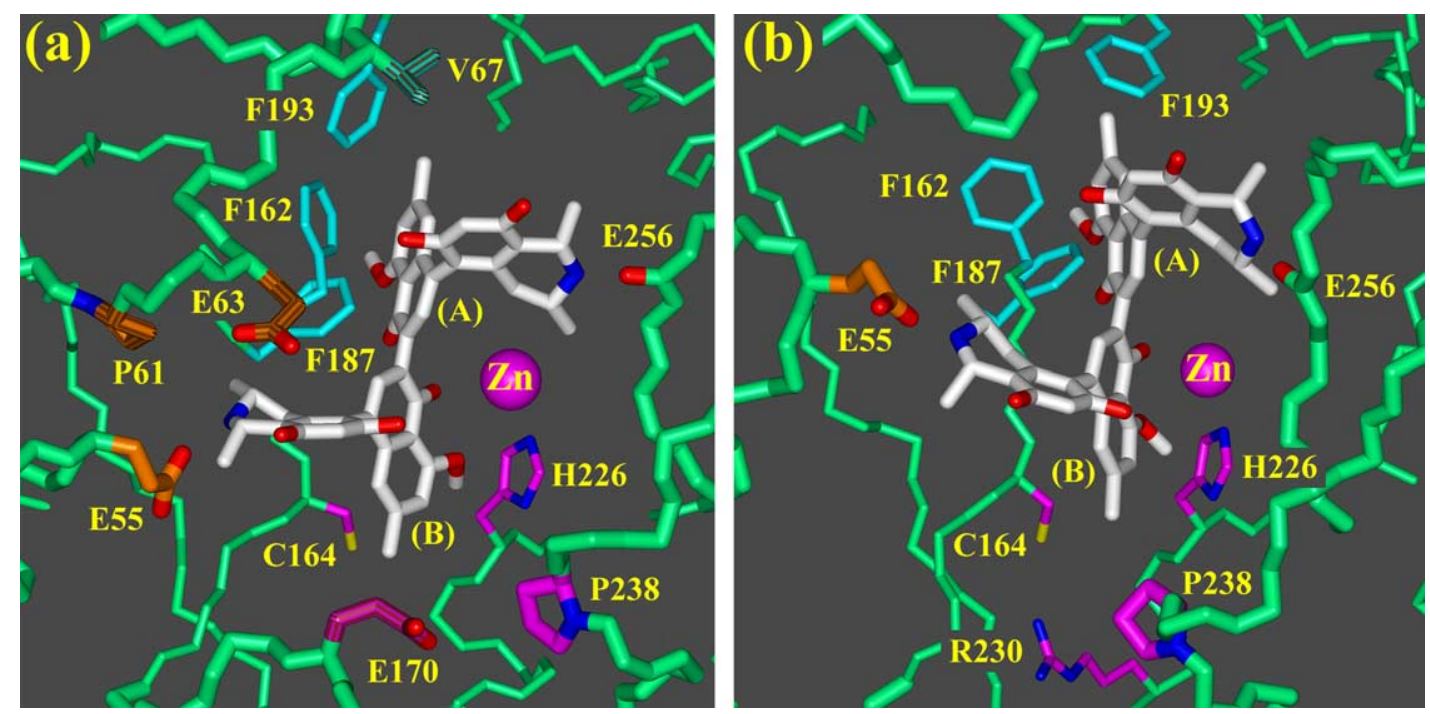

Figure 4. (a) Michellamine B docked in the molecular dynamics structure for the 3BTA BoNT/A LC. All colors and stick thickness are as described in the caption for Figure 3a; (b) michellamine B docked in the molecular mechanics refined X-ray crystal structure of the 3BTA BoNT/A LC. All colors and stick thickness are the same as described in Figure 3a. A comparison of (a) and (b) shows that their are more favorable electrostatic and hydrophobic contacts between michellamine B and the BoNT/A LC dynamics structure, and that these interactions are the result of loop reorientations toward the substrate binding cleft. 
Residues in the previously identified polar contact region (composed of residues E55, Q161, E163, K165, and $\mathrm{R} 176^{24}$ ) for inhibitors also remained consistent over the course of the dynamics trajectory. However, loop 1 reorientation partially covers this contact region, leaving it less solvent accessible, and also brings new contact residues into association with this binding region.

Models of Q2-15 docked in both the BoNT/A LC dynamics structure (Fig. 3a) and the mechanics refined $\mathrm{X}$-ray structure (Fig. 3b) indicate that the inhibitors ionizable secondary nitrogen engages in a hydrogen bond with the side-chain carboxylate of E163. Furthermore, due to loop 1 reorientation in the dynamics structure, the inhibitor's secondary nitrogen also engages in an ion-dipole interaction with the backbone carbonyl of K65 (Fig. 3a). For comparison, the ionizable nitrogen of the michellamine B tetrahydro-isoquinoline moiety attached to naphthalene ring $B$ engages in a hydrogen bond with the side-chain carboxylate of residue E55, and is also in close proximity to the side chain carboxylate of E163, in both the dynamics and molecular mechanics refined models of 3BTA. Furthermore, in the 3BTA dynamics structure (Fig. 4a), loop 1 reorientation resulted in additional residue contacts with the 1,3dimethyl-1,2,3,4-tetrahydro-isoquinoline-6,8-diol moiety attached to naphthalene ring B. Specifically, the side-chain carboxylate of E63 is located near the ionizable nitrogen of the 1,3-dimethyl-1,2,3,4-tetrahydro-isoquinoline-6,8-diol moiety, and engages in a hydrogen bond with the 8-hydroxyl substituent, while the moieties 3-methyl substituent engages in a hydrophobic interaction with the P61 pyrrolidine (Fig. 4a).

In general, Q2-15 and michellamine B, although structurally very different, engage in similar contacts in the BoNT/A LC substrate binding cleft-regardless of the enzyme model that is used. These two compounds also share comparable pharmacophoric features, ${ }^{24}$ which would seem to explain their comparable inhibitory potency. Furthermore, analyses of inhibitors docked in the dynamics structures, versus mechanics refined Xray crystal structures of both $1 \mathrm{E} 1 \mathrm{H}$ and $3 \mathrm{BTA}$, indicate that the models obtained from the dynamics simulations provide additional inhibitor contacts that may be important for inhibitor binding. In particular, additional hydrophobic contacts near subsite 1, additional contacts at the polar contact region, and a reduction in the solvent accessibility at subsite 2 (following loop 2 reorientation toward the substrate binding cleft) all seem to suggest that possible loop reorientations toward the BoNT/A LC substrate binding cleft may be important for understanding inhibitor/substrate binding, and consequently, for the future development of small molecule inhibitors.

\subsection{Potential silver ion binding sites}

We have also reported that silver ion inhibits BoNT/A LC metalloprotease activity $(100 \%$ inhibition at $\geqslant 5 \mu \mathrm{M}$ ) without displacing the catalytic zinc ion or causing enzyme denaturation. ${ }^{24}$ Observed loop 1 movement toward the substrate binding cleft may aid in par-

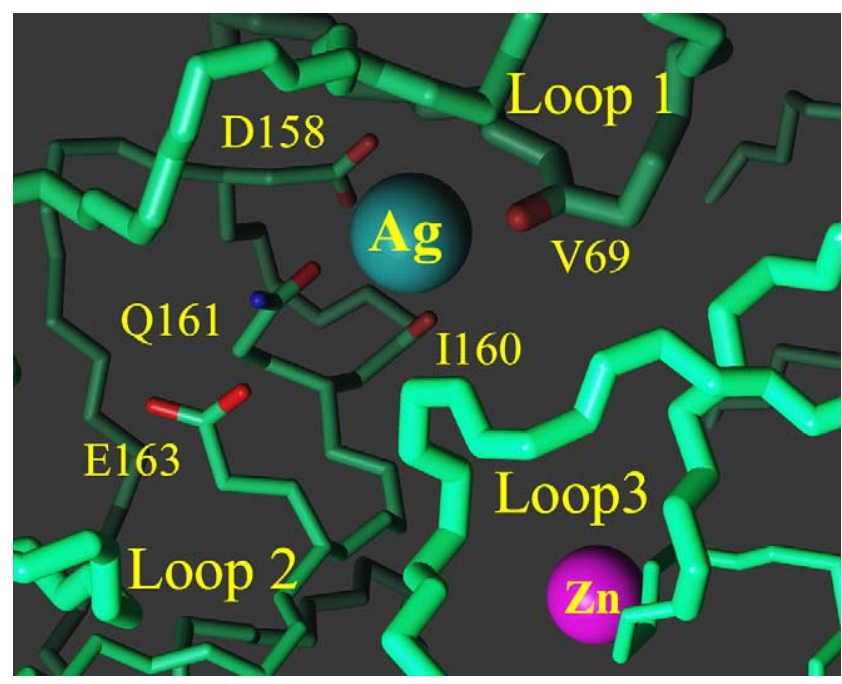

Figure 5. A proposed binding site for silver ion in the dynamics structure of the 3BTA BoNT/A LC. Oxygen atoms are red and nitrogen atoms are blue. The silver ion is shown as a light blue sphere and the zinc ion is a magenta sphere. All other atoms are green. Loops 1,2 , and 3 are shown in thicker stick. Loop 1 reorientation partially shields the polar contact region from solvent, creating a pocket that may potentially trap a silver ion.

tially explaining these results. In particular, Figure 5 shows how loop 1 reorientation in the 3BTA dynamics structure creates a pocket that could potentially trap a silver ion. In Figure 5, a silver ion is shown engaging in an ionic interaction with the side-chain carboxylate of D158, and ion-dipole interactions with the side-chain amide carbonyl of Q161, and the backbone carbonyl oxygens of V69 and I160. In addition to the above contacts, the silver ion might also interact with other residues in the polar contact region, including E163. It should also be noted that binding subsite 2 may provide a potential silver ion contact region, with the ion coordinated to the C164 side-chain thiol, and engaging in electrostatic interactions with surrounding residues $\mathrm{H} 226$ and E260. Interestingly, there are no other regions in or near the substrate binding cleft that would provide similar clusters of residues for silver ion 'trapping'.

\subsection{Refinement of the BoNT/A LC inhibitor pharmacophore}

Figure 6 shows the components of our pharmacophore for BoNT/A LC inhibition. ${ }^{24}$ Components $\mathbf{A}$ and $\mathbf{B}$ in Figure 6 represent two planar moieties, one of which contains a heteroatom that may engage in an interaction with the enzyme's catalytic zinc, or potentially replace the water used by the zinc engine during substrate lysis. Examples of components $\mathbf{A}$ and $\mathbf{B}$ include the two-quinoline rings of Q2-15 (Figs. 1 and 3a and b) and the two naphthalene rings of michellamine B (Figs. 1 and $4 \mathrm{a}$ and b). In Figure 6, pharmacophore components $\mathbf{C}$ and $\mathbf{D}$ are two hydrophobic substituents, which are predicted to interact with subsites 1 and 2 in the substrate binding cleft, respectively. Examples of components $\mathbf{C}$ and $\mathbf{D}$ include the two chloro substituents of Q2-15 and the methoxy substituents (attached to the naphthalene 


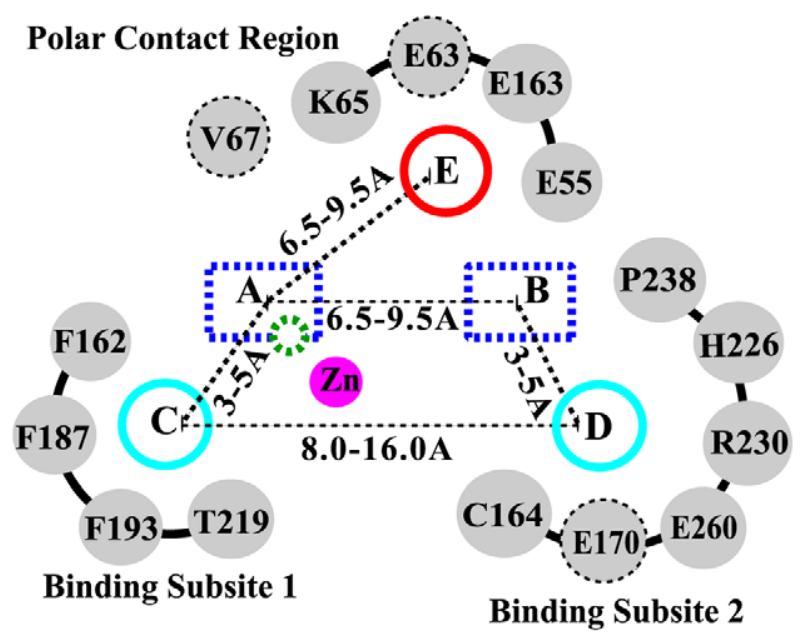

Figure 6. Refined pharmacophore for BoNT/A LC inhibition. Planar components $\mathbf{A}$ and $\mathbf{B}$ are blue dashed rectangles. The dashed circle in plane A represents a heteroatom. Hydrophobic components $\mathbf{C}$ and $\mathbf{D}$ are shown as light blue circles. The positive ionizable component $\mathbf{E}$ of the pharmacophore is shown as a red circle. Residues that remained consistent when docking inhibitors in predicted binding subsites of both dynamics and molecular mechanics 'only' refined models are shown as gray spheres. Residues E63, V67, and E170 are shown as a gray spheres with dashed black boarders - to indicate that these amino acids were found to participate when docking inhibitors in dynamics structures.

rings) of michellamine B. Finally, the polar, ionizable pharmacophore component $\mathbf{E}$ (Fig. 6) is hypothesized to either engage in electrostatic or water mediated interactions with residues in the polar contact region. Examples of component $\mathbf{E}$ include the secondary nitrogen of Q2-15 and the secondary nitrogen of one of the tetrahydro-isoquinolines of michellamine B.

Comparisons between inhibitors docked in molecular dynamics models (Figs. 3a and 4a) and inhibitors docked in molecular mechanics refined models (Figs. $3 \mathrm{~b}$ and $4 \mathrm{~b}$ ) indicate that inhibitor residue contacts in binding subsites 1 and 2, and the polar contact region, remain relatively consistent during the dynamic movement of BoNT/A LC. Moreover, our analyses indicate that loop reorientations may bring additional residues into play, which may serve to facilitate inhibitor binding. Based on these observations, the BoNT/A LC inhibitor pharmacophore has been refined via the addition of residues that are predicted to interact with specified pharmacophore components (Fig. 6). Table 2 provides an overview of the torsional angle ranges of residues shown in Figure 6. In agreement with comparable dynamic motion observed for the two BoNT/A LCs, torsional ranges for the indicated contact residues also follow similar trends.

\section{Conclusions}

These studies have, for the first time, used molecular dynamics to explore conformationally available binding contacts for our BoNT/A LC inhibitors. ${ }^{24}$ Results from dynamics simulations performed on two separate

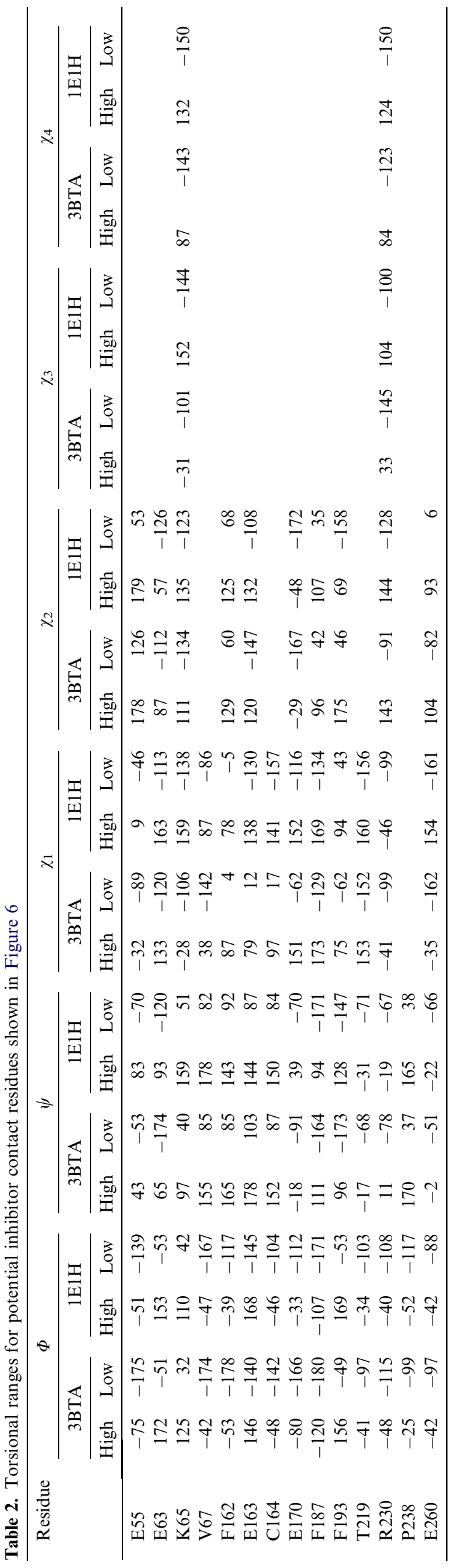


BoNT/A LCs indicated that the $\alpha$-helices and $\beta$-sheets of this enzyme remain stable over time, but that conformational flexibility in surface loops surrounding the substrate binding cleft may affect inhibitor binding. Specifically, our results show that surface loops 1 and 2 may reorient, thereby creating additional residueinhibitor contacts, as well as serving to decrease the solvent accessibility of the substrate binding cleft. Finally, these studies have provided a unique opportunity to reexamine BoNT/A LC inhibitor binding, and to further refine a previously proposed pharmacophore for inhibition via the inclusion of potential binding subsite contacts.

\section{Acknowledgements}

The research described herein was sponsored by the US Army Medical Research and Material Command Research Plan \# 02-4-3U-057 and IAA \# Y3-CM-100505 (MRMC and NCI). We acknowledge the National Cancer Institute for the allocation of computing time and staff support at the Advanced Biomedical Computing Center of the Frederick Cancer Research and Development Center. The contents of this publication do not necessarily reflect the views or policies of the Department of Health and Human Services, nor does the mention of trade names, commercial products, or organizations imply endorsement by either the US Government or the US Army.

\section{References and notes}

1. Foran, P. G.; Davletov, B.; Meunier, F. A. Trends Mol. Med. 2003, 9, 291.

2. Arnon, S. S.; Schechtler, R.; Inglesby, T. V.; Henderson, D. A.; Bartlett, J. G.; Ascher, M. S.; Eitzen, E.; Fine, A. D.; Hauer, J.; Layton, M.; Lillibridge, S.; Osterholm, M. T.; O'Toole, T.; Parker, G.; Perl, T. M.; Russell, P. K.; Swerdlow, D. L.; Tonat, K. J. Am. Med. Assoc. 2001, 285, 1059.

3. Rossetto, O.; Seveso, M.; Caccin, P.; Schaivo, G.; Montecucco, C. Toxicon 2001, 39, 27.

4. Gui, D.; Rossi, S.; Runfola, M.; Magalini, S. C. Aliment. Pharmacol. Ther. 2003, 18, 1 .

5. O'Brien, C. F. Clin. J. Pain 2002, 18, S182.

6. Sheean, G. Curr. Pain Headache Rep. 2002, 6, 460.

7. Kessler, K. R.; Benecke, R. Neurotoxicology 1997, 18, 761.

8. Said, S.; Meshkinpour, A.; Carruthers, A.; Carruthers, J. Am. J. Clin. Dermatol. 2003, 4, 609.

9. Glogau, R. G. Clin. J. Pain 2002, 18, S191.

10. Sposito, M. M. Plast Reconstr. Surg. 2002, 110, 601.

11. Franz, D. R.; Jahrling, P. B.; Friedlander, A. M.; McClain, D. J.; Hoover, D. L.; Byrne, W. R.; Pavlin, J. A.; Christopher, G. W.; Eitzen, E. M., Jr. J. Am. Med. Assoc. 1997, 278, 399.
12. Meunier, F. A.; Lisk, G.; Sesardic, D.; Dolly, J. O. Mol. Cell. Neurosci. 2003, 22, 454.

13. Lacy, D. B.; Stevens, R. C. J. Mol. Biol. 1999, 291, 1091.

14. Turton, K.; Chaddock, J. A.; Acharya, K. R. Trends Biochem. Sci. 2002, 27, 552.

15. Singh, B. R. Nat. Struct. Biol. 2000, 7, 617.

16. Lacy, D. B.; Tepp, W.; Cohen, A. C.; DasGupta, B. R.; Stevens, R. C. Nat. Struct. Biol. 1998, 5, 898.

17. Dolly, O. Headache 2003, 43, S16.

18. Binz, T.; Blasi, J.; Yamasaki, S.; Baumeister, A.; Link, E.; Sudhof, T. C.; Jahn, R.; Niemann, H. J. Biol. Chem. 1994, $269,1617$.

19. Schiavo, G.; Benfenati, F.; Poulain, B.; Rossetto, O.; Polverion de Laureto, P.; DasGupta, B. R.; Montecucco, C. Nature 1992, 359, 832.

20. Schiavo, G.; Rossetto, O.; Catsicas, S.; Polveriono de Laureto, P.; DasGupta, B. R.; Benfenati, F.; Montecucco, C. J. Biol. Chem. 1993, 268, 23784.

21. Schiavo, G.; Shone, C. C.; Rossetto, O.; Alexander, F. C.; Montecucco, C. J. Biol. Chem. 1993, 268, 11516.

22. Schiavo, G.; Malizio, C.; Trimble, W. S.; Polveriono de Laureto, P.; Milan, G.; Sugiyama, H.; Johnson, E. A.; Montecucco, C. J. Biol. Chem. 1994, 269, 20213.

23. Blasi, J.; Chapman, E. R.; Yamasaki, S.; Binz, T.; Niemann, H.; Jahn, R. EMBO J. 1993, 12, 4821.

24. Burnett, J. C.; Schmidt, J. J.; Stafford, R. G.; Panchal, R. G.; Nguyen, T. L.; Hermone, A. R.; Vennerstrom, J. L.; McGrath, C. F.; Lane, D. J.; Sausville, E. A.; Zaharevitz, D. W.; Gussio, R.; Bavari, S. Biochem. Biophys. Res. Commun. 2003, 310, 84.

25. Segelke, B.; Knapp, M.; Kadkhodayan, S.; Balhorn, R.; Rupp, B. Proc. Natl. Acad. Sci. U.S.A. 2004, 101, 6888.

26. Li, L.; Singh, B. R. J. Toxicol., Toxin Rev. 1999, 18, 95.

27. Kadkhodayan, S.; Knapp, M. S.; Schmidt, J. J.; Fabes, S. E.; Rupp, B.; Balhorn, R. Protein Expr. Purif. 2000, 19, 125.

28. Giannakakou, P.; Gussio, R.; Nogales, E.; Downing, K. H.; Zaharevitz, D.; Bollbuck, B.; Poy, G.; Sackett, D.; Nicolaou, K. C.; Fojo, T. Proc. Natl. Acad. Sci. U.S.A. 2000, 97, 2904.

29. Panchal, R. G.; Hermone, A. R.; Nguyen, T. L.; Wong, T. Y.; Schwarzenbacher, R.; Schmidt, J. J.; Lane, D.; McGrath, C. F.; Turk, B. E.; Burnett, J. C.; Aman, M. J.; Little, S.; Sausville, E. A.; Zaharevitz, D. W.; Cantley, L. C.; Liddington, R. C.; Gussio, R.; Bavari, S. Nat. Struct. Biol. 2004, 11, 1.

30. Schmidt, J. J.; Stafford, R. G. Appl. Environ. Microbiol. 2003, 69, 297.

31. Schmidt, J. J.; Bostian, K. A. J. Protein Chem. 1997, 16, 19.

32. Ahmed, S. A.; Byrne, M. P.; Jensen, M.; Hines, H. B.; Brueggemann, E.; Smith, L. A. J. Protein Chem. 2001, 20, 221.

33. Ahmed, S. A.; McPhie, P.; Smith, L. A. Biochemistry 2003, 42, 12539.

34. Hanson, M. A.; Stevens, R. C. Nat. Struct. Biol. 2000, 7, 687.

35. Swaminathan, S.; Eswaramoorthy, S. Nat. Struct. Biol. 2000, 7, 693.

36. Schmidt, J. J.; Stafford, R. G.; Bostian, K. A. FEBS Lett. 2002, 532, 423. 